\title{
Anti-arthritic Effects of Oplopanax elatus in a Rat Model of Rheumatoid Arthritis (Adjuvant-induced Arthritis)
}

\author{
Ki Sun Kwon ${ }^{1}$, Hyun Lim ${ }^{1}$, Yong Soo Kwon ${ }^{1}$, Hye Ri Choi ${ }^{1}$, Myong Jo Kim ${ }^{2}$, \\ Ji Hye Yoo ${ }^{3}$, Nam Ho Yoo ${ }^{2}$, and Hyun Pyo Kim ${ }^{1, *}$ \\ ${ }^{1}$ College of Pharmacy, Kangwon National University, Chuncheon 24341, Korea \\ ${ }^{2}$ Department of Bio-Resource Sciences, Kangwon National University, Chuncheon 24341, Korea \\ ${ }^{3}$ Bioherb Research Institute, Kangwon National University, Chuncheon 24341, Korea
}

\begin{abstract}
The stems of Oplopanax elatus (OE) have long been used to treat inflammatory disorders in herbal medicine, and in the previous investigation, OE was found to possess anti-inflammatory activity in lipopolysaccharide-treated macrophages, RAW 264.7 cell. OE reduces inducible nitric oxide (NO) synthaseinduced NO production, and interferes with mitogen-activated protein kinase activation pathways. In the present study, the pharmacological action of the water extract of $\mathrm{OE}$ was examined to establish anti-arthritic action, using a rat model of adjuvant-induced arthritis (AIA). The water extract of OE administered orally inhibited AIAinduced arthritis at $(100-300) \mathrm{mg} / \mathrm{kg} /$ day. The paw edema was significantly decreased, in combination with reduced production of pro-inflammatory cytokines. The action mechanism includes an inhibition of MAPKs/ nuclear transcription factor- $\kappa \mathrm{B}$ activation. These new findings strongly suggest that $\mathrm{OE}$ possesses anti-arthritic action, and may be used as a therapeutic agent in inflammation-related disorders, particularly in arthritic condition.
\end{abstract}

Keywords - Oplopanax elatus, rheumatoid arthritis, adjuvant-induced arthritis, mitogen-activated protein kinase

\section{Introduction}

Rheumatoid arthritis (RA) is an immunological disease that mainly affects joint spaces, finally leading to the disabled state. In the joints, many inflammatory cells, including neutrophils and macrophages, as well as synovial fibroblasts, participate in the disease process, producing inflammatory characteristics: fever, redness, pain, and deformation of bone structures. The long-term treatment with currently used drugs, such as nonsteroidal anti-inflammatory drugs (NSAIDs) and steroidal antiinflammatory drugs (SAIDs), often produces serious sideeffects. ${ }^{1,2}$ Several disease modifying drugs are also used, but the low effectiveness and side-effects may curtail their wide and safe use. Recently, biological drugs, such as monoclonal antibody against interleukin-6 (IL-6), ${ }^{3}$ are being prescribed, but they also have some limitations to cure the disorders. On the other hand, in some cases, especially in the period of initial phases of RA and/or the conditions showing mild symptoms, plant-derived drugs

\footnotetext{
*Author for correspondence

H. P. Kim, College of Pharmacy, Kangwon National University, Chuncheon 24341, Korea

Tel: +82-33-250-6915; E-mail: hpkim@kangwon.ac.kr
}

may be a beneficial alternative to alleviate symptoms, with less side-effect in long-term use. Under this notion, several plant products are being used as main therapeutics, as well as supplementary treatment. ${ }^{4}$

Oplopanax elatus Nakai (Araliaceae) is a plant that is found in the northern parts of Korea and China. ${ }^{5}$ This plant has been used in traditional Chinese medicine to treat chronic fatigue syndrome, pain, diabetes, and hypertension. ${ }^{6,7}$ In Korea, the roots and stems of $O$. elatus are mostly used for alleviating the symptoms of RA. Some anti-inflammatory activities of this plant material have previously been reported. Oral administration of the ethanol extract of $O$. elatus $(10 \mathrm{~g} / \mathrm{kg})$ inhibited paw edema induced by formalin and dextran, as well as cotton-pelletinduced granuloma in rats. ${ }^{7,8}$ Recently, we have evaluated the in vitro anti-inflammatory action of the water extract of $O$. elatus (unpublished data). However, although $O$. elatus has been potentially used as anti-arthritic agent, in vivo anti-arthritic action has not been proved to date. Therefore in the present investigation, the in vivo antiarthritic activity of the water extract of $O$. elatus has been evaluated to provide the scientific basis of its use, and to find therapeutic potential for human use as a herbal drug. 


\section{Experimental}

Chemicals - Prednisolone was purchased from SigmaAldrich Inc. (St. Louis, MO, USA). Protein assay kit was a product of Bio-Rad Lab. (Hercules, CA). Rat IL-1 $\beta$ and IL-6 ELISA kits were purchased from R\&D systems (Minneapolis, MN, USA) and BD bioscience (San Diego, CA, USA), respectively. Rat tumor necrosis factor- $\alpha$ (TNF- $\alpha$ ) ELISA kit was obtained from eBioscience (San Diego, CA, USA). Rat C-reactive protein (CRP) ELISA kit was purchased from Invitrogen (San Diego, CA, USA). Alanine aminotransferase (ALT) activity assay kit and aspartate aminotransferase (AST) activity assay kit were purchased from Biovision (Milpitas, CA, USA). Antibodies against MAPKs, nuclear transcription factor$\kappa \mathrm{B}(\mathrm{NF}-\kappa \mathrm{B})$ and $\mathrm{c}-J u n$ were purchased from Cell Signaling Technologies (Danvers, MA, USA). $\beta$-Actin antibody was purchased from Bethyl Laboratories, Inc. (Montgomery, TX, USA). Lamin B1 antibody was purchased from Bioworld technology, Inc. (Minneapolis, MN, USA). QuantiTect primer sets for IL-1 $\beta$ (QT 00181657), IL-6 (QT00182896), TNF- $\alpha$ (QT00178717) and $\beta$-actin (QT00193473) were purchased from Qiagen (Melbourne, Australia). Hematoxylin and eosin solution were bought from Merck (Darmstadt, Germany).

Animal - Wistar rats (male, $80-100 \mathrm{~g}$, specific pathogenfree) were obtained from Orient Bio Inc. (Seongnam, Korea). The animals were maintained in an animal facility (KNU) at $20-22{ }^{\circ} \mathrm{C}$ under $40-60 \%$ relative humidity and a $12 \mathrm{~h} / 12 \mathrm{~h}$ (light/dark) cycle. The animal experiment was approved by the local committee for animal experimentation, KNU (KW-170717-1). The ethical guideline described in the Korean Food and Drug Administration guide for the care and use of laboratory animals was followed throughout the experiments.

Preparation of the water extract of the stems of $O$. elatus (WOE) - The stems of O. elatus (OE) were collected in the Hwa-Ack mountain in 2012 and authenticated by one of the authors, Dr. Y. S. Kwon. The dried stems of $O$. elatus $(8 \mathrm{~kg})$ was extracted in boiling water for 4 hours by refluxing. After filtering, the residue was freeze-dried to give the water extract of OE (WOE, $450 \mathrm{~g}$ ). WOE has been found to contain syringin $(214.0 \mu \mathrm{g} / \mathrm{g}$ dried plant material) for a standard constituent (unpublished data).

Adjuvant-induced arthritis (AIA)-Experimental arthritis was induced by subplantar injection of heat-killed Mycobacterium butyricum (Benton, Dickinson and Company, Sparks, MD, USA) suspended in mineral oil as previously described. ${ }^{9}$ Mycobacterium butyricum dissolved in mineral oil $(0.6 \mathrm{mg} / 0.1 \mathrm{~mL})$ was injected into the right hind paw of Wistar rats $(0.6 \mathrm{mg} / \mathrm{rat}, n=6)$. Prednisolone was used as a reference drug. The animals were randomly divided into five groups: control (C), adjuvant-treated control (AC), adjuvant + WOE $300 \mathrm{mg} / \mathrm{kg} /$ day (AW300), adjuvant + WOE $100 \mathrm{mg} / \mathrm{kg} /$ day (AW100), and adjuvant + prednisolone $5 \mathrm{mg} / \mathrm{kg} /$ day (AP5). WOE and prednisolone dissolved in $0.3 \%$ CMC were orally administered $(0.3$ $\mathrm{mL} /$ rat) daily for 21 days. The right hind paw weights of rats were measured every 2 days using plethysmometer (Ugo Basile, Italy). At 21 days after adjuvant injection, animals were sacrificed, blood was collected from the heart, and serum was separated after centrifugation at 5,000 rpm for $10 \mathrm{~min}$ at $4{ }^{\circ} \mathrm{C}$. Enzyme-linked immunosorbent assay (ELISA) was performed on the separated serum according to the protocols enclosed in the ELISA kit $(n=6)$. In order to confirm the inflammation and cartilage damage, the right paws were obtained, and after demineralization with $10 \%$ ethylenediaminetetraacetic acid (EDTA), they were fixed with paraffin to make a slice with a thickness of $5 \mu \mathrm{m}(n=3)$. Tissue sections were stained with hematoxylin \& eosin, and observed with an optical microscope.

Real-time PCR analysis - The expression levels of proinflammatory cytokines were measured by real-time PCR from the remaining paws of the first experiment above $(n=3)$. The tissues were homogenized in $1 \mathrm{~mL}$ of Trizol (Favorgen Biotech Corp., Taiwan). Total RNA was extracted with chloroform/isopropanol and purified with RNA clean-up kit (MACHEREY-NAGEL GmbH\&Co, Düren, Germany). cDNA was synthesized with ReverTra Ace qPCR RT Master Mix and gDNA Remover (Toyobo, Osaka, Japan) and then proceeded to real-time PCR cycling using THUNDERBIRD SYBR qPCR mix (Toyobo, Osaka, Japan) on C1000 Touch Thermal Cycler (Bio-rad Lab., Hercules, CA, USA). The expression levels of proinflammatory cytokines mRNA were normalized to that of $\beta$-actin.

Western blotting - For obtaining the paw tissues for Western blotting analysis, parallel experiment following the same treatment regimen as above was carried out $(n=$ 6). After 21 days, the right hind paws were obtained $(n=$ 3) and they were homogenized. From these tissue homogenate, Western blot was performed. To measure the degree of MAPKs activation, the tissues were homogenized in Pro-prep solution (iNtRON Biotechnology, Seongnam, Korea) containing $1 \mathrm{mM}$ phenylmethanesulfonyl fluoride (PMSF), $1 \mathrm{mM}$ sodium fluoride $(\mathrm{NaF})$ and $1 \mathrm{mM}$ sodium orthovanadate, and centrifuged at $12,000 \mathrm{rpm}$ for $10 \mathrm{~min}$ at $4{ }^{\circ} \mathrm{C}$. The supernatant was quantified with Bradford solution (Bio-rad Lab., Hercules, CA, USA) and electro- 
phoresis was performed in $10 \%$ sodium dodecyl sulfate (SDS) polyacrylamide gels. On the other hand, for measuring the activation levels of transcription factors, tissues $(n=3)$ were homogenized in $400 \mu \mathrm{L}$ of buffer A [10 mM 4-(2-hydroxyethyl)-1-piperazineethanesulfonic acid (HEPES), pH 7.9, $10 \mathrm{mM} \mathrm{KCl}, 0.1 \mathrm{mM}$ EDTA, $1 \mathrm{mM}$ 1,4-dithiothreitol (DTT), $0.5 \mathrm{mM}$ PMSF, $1 \mathrm{mM}$ $\mathrm{NaF}, 1 \mathrm{mM}$ sodium orthovanadate] and added $25 \mu \mathrm{L}$ of $10 \% \mathrm{NP}-40$, the solution was vortexed and centrifuged at $5,000 \mathrm{rpm}$ for $2 \mathrm{~min}$ at $4{ }^{\circ} \mathrm{C}$. And the nuclear pellet was vigorously vortexed in buffer B (20 mM HEPES, $\mathrm{pH} 7.9$, $0.4 \mathrm{M} \mathrm{NaCl}, 1 \mathrm{mM}$ EDTA, $1 \mathrm{mM}$ DTT, $1 \mathrm{mM}$ PMSF, $1 \mathrm{mM} \mathrm{NaF}, 1 \mathrm{mM}$ sodium orthovanadate) and centrifuged at $13,000 \mathrm{rpm}$ for $10 \mathrm{~min}$ at $4{ }^{\circ} \mathrm{C}$. After the supernatant

(A)

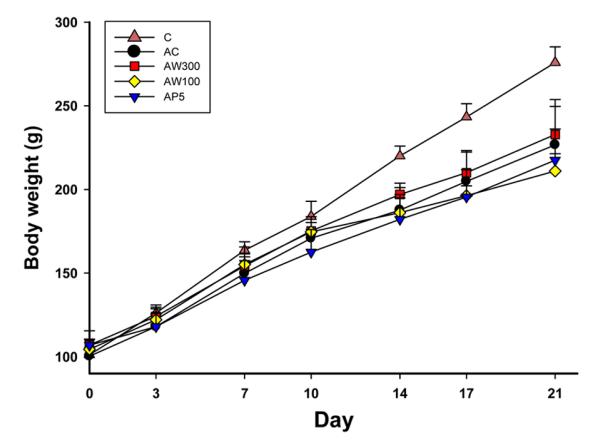

(C)

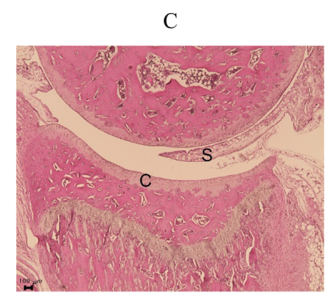

AW300
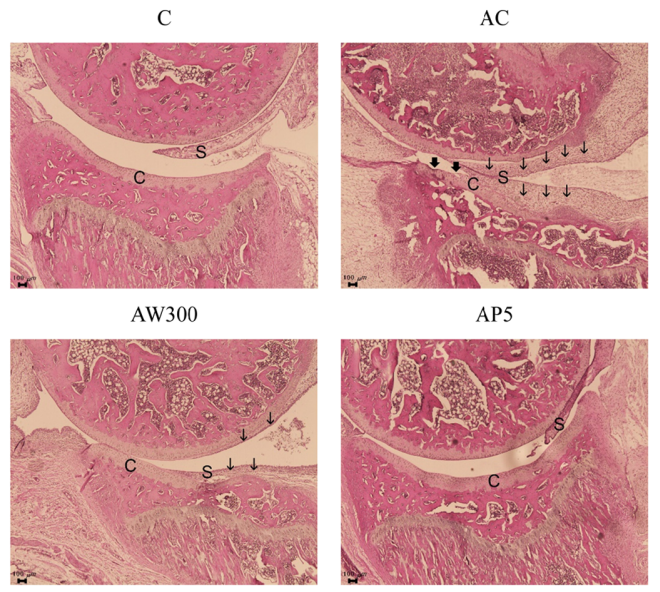

AP5

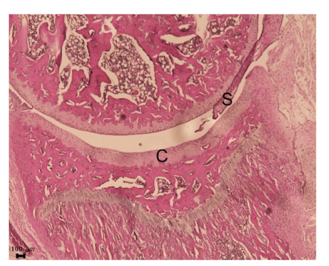

was quantified with Pierce BCA protein assay kit (Thermo Scientific, Rockford, IL, USA) and electrophoresis was performed in $10 \%$ SDS gels, the proteins were transferred to polyvinylidene difluoride (PVDF) membranes. The transferred PVDF membranes were blocked with 5\% skim milk in Tris-buffered saline with $0.05 \%$ Tween 20 (TBST) for $1 \mathrm{~h}$ at room temperature and incubated with primary antibody (1:1000 in 5\% skim milk in TBST) for overnight at $4{ }^{\circ} \mathrm{C}$. After membranes were incubated with secondary antibody diluted $1: 5000$ in 5\% skim milk in TBST for $1 \mathrm{~h}$ at room temperature, the bound antibodies were detected by enhanced chemiluminescence (West Femto Luminol/Enhancer solution, Thermo Scientific, Rockford, IL, USA).

(B)

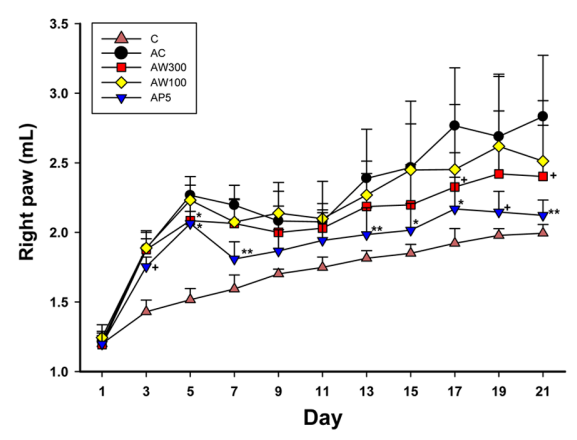

(D)
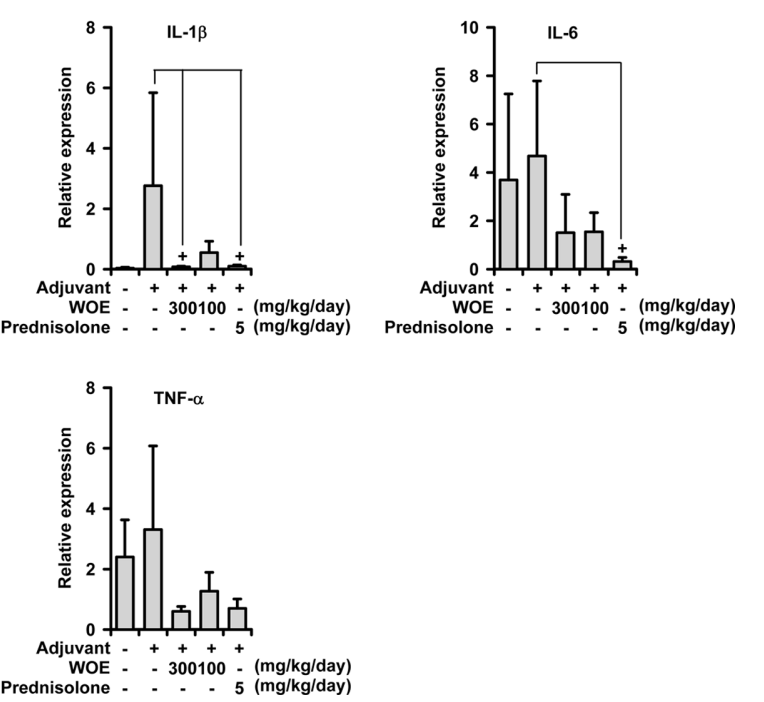

Fig. 1. Inhibition of arthritic inflammation by WOE on adjuvant-induced arthritis in rats. (A) Body weight change $(n=6)$, (B) Changes of rat right hind paw volume $(n=6), \%$ inhibition $=$ [paw volume of AC - (paw volume of test sample - paw volume of C)] / (paw volume of AC - paw volume of C) $\times 100$, (C) Histologic observation of ankle joint of right hind paw, One set of three samples per group is represented here., Hematoxylin and eosin staining, $\times 40, \mathrm{C}$ (cartilage), S (synovial tissue), cartilage destruction $(\rightarrow)$, cellular infiltration $(\rightarrow)$, (D) Gene expression of pro-inflammatory molecules (IL-1 $\beta$, IL-6, and TNF- $\alpha$ ) in right hind paw tissue $(n=3)$, The paw tissues were homogenized, and total RNA were extracted, followed by real-time PCR analysis., Control (C), adjuvant-treated control (AC), adjuvant + WOE $300 \mathrm{mg} / \mathrm{kg} /$ day (AW300), adjuvant + WOE $100 \mathrm{mg} / \mathrm{kg} /$ day (AW100), adjuvant + prednisolone $5 \mathrm{mg} / \mathrm{kg} / \mathrm{day}(\mathrm{AP} 5),{ }^{+} P<0.1,{ }^{*} P<$ $0.05, * * P<0.01$, significantly different from AC. 
Statistical analysis - All experimental data were represented as arithmetic mean $\pm \mathrm{SD}$. One way ANOVA followed by Dunnett's test was used to determine the statistical significance. For the analysis, IBM SPSS Statistics version 22 (IBM, Armonk, NY, USA) was used.

\section{Results and Discussion}

To induce arthritic inflammation, heat-killed mycobacterium butyricum, an adjuvant, was injected into the right hind paw of the rats $(n=6)$. For anti-arthritic effects of WOE, WOE and prednisolone were orally administered 1 hour before the adjuvant injection, and the test compounds were further administered orally once daily for 3 weeks. At last day, WOE and prednisolone were orally administered, and two hours later, paw volume of the right hind paw was measured, and thereafter, the animals were sacrificed. For three weeks, the body weight of control group (C) increased over $150 \mathrm{~g}$, while the body weights of adjuvant-treated groups (AC, AW100, AW300 and AP5) increased less than $120 \mathrm{~g}$ (Fig. 1A). No significant change was observed in the body weight increase between $\mathrm{AC}$ and the test compounds-treated groups (AW100, AW300 and AP5). The paw edema increased initially to $3-5$ days, and started to further

(A)
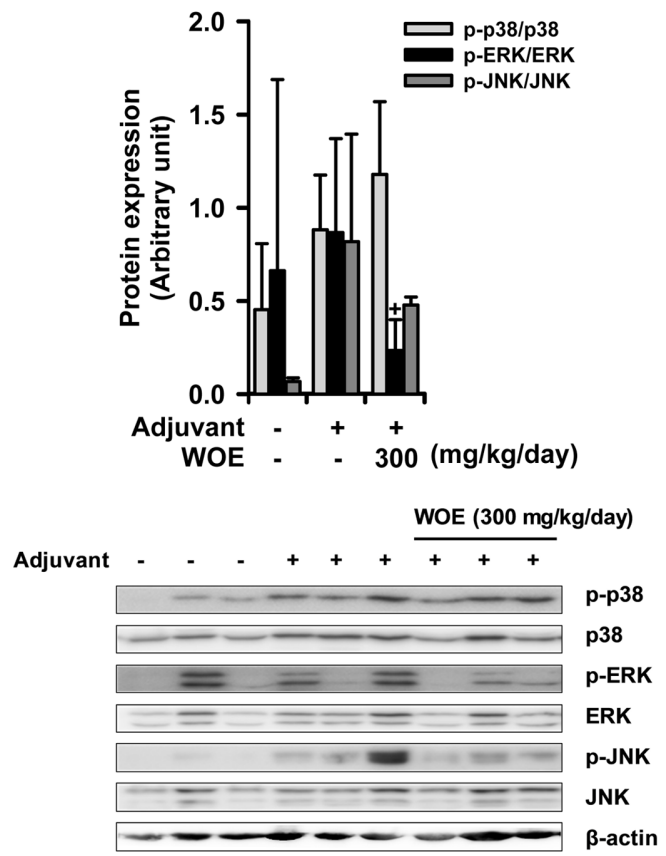

increase around 10 days after adjuvant injection, with arthritic inflammation, as expected. At 21 days after adjuvant injection, the net increase of paw volume of the right hind paw in $\mathrm{AC}$ was approximately $0.8 \pm 0.4 \mathrm{~mL}$. The paw edema of the rats at 21 days after the injection of adjuvant was significantly inhibited in AW300 and AP5 by $51.3 \%$ and $84.7 \%$, respectively (Fig. 1B).

When histologically observed, the paws of AC clearly showed severe synovial hyperplasia, inflammatory cell infiltration and cartilage destruction as typical markers of rheumatoid arthritis (Fig. 1C). WOE treatment (AW300) strongly and notably reduced these inflammatory responses, confirming the anti-arthritic action of WOE.

Finally, total RNA was isolated by homogenization of the paw tissues, and the expression levels of proinflammatory cytokines were measured by real-time PCR $(n=$ 3 ). In the case of IL-1 $\beta$, the amount of mRNA expression was increased in AC compared to the control (C) group, and AW300 and AP5 almost completely abolished the degree of expression by $98.4 \%$ and $97.5 \%$ reduction, respectively (Fig. 1D). However, IL-6 and TNF- $\alpha$ mRNA expressions were not increased notably in $\mathrm{AC}$, compared to the control group, indicating that IL- 6 and TNF- $\alpha$ might not be critically involved in producing arthritic inflammation in the paws of AIA rats. Nevertheless, they

(B)
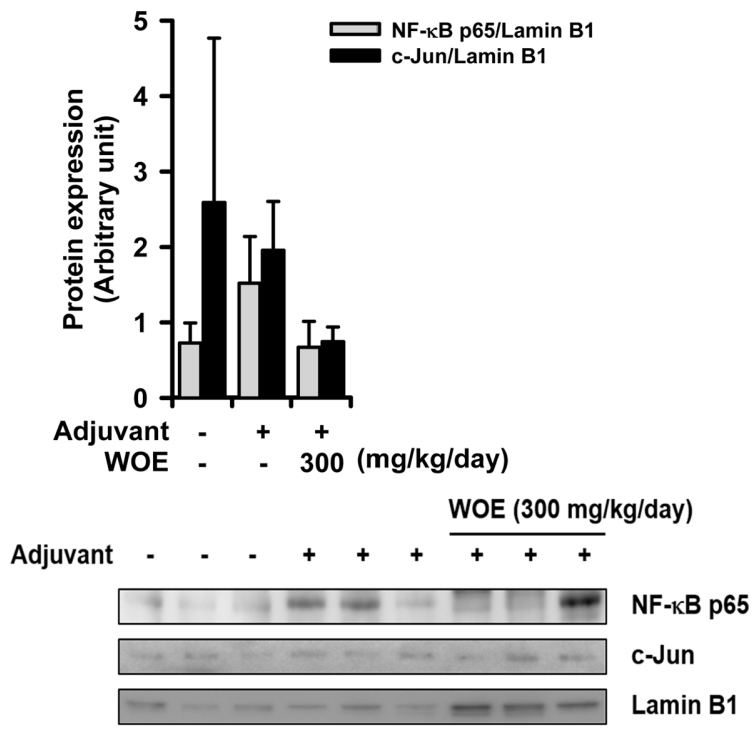

Fig. 2. Effects of WOE on the signaling pathways of arthritic inflammation in adjuvant-induced arthritis rats. (A) Effects of WOE (300 $\mathrm{mg} / \mathrm{kg} /$ day) on MAPK pathways (p38 MAPK, ERK, and JNK), (B) Effects of WOE (300 mg/kg/day) on nuclear transcription factor activation (NF-KB p65 and c-Jun), Western blot analysis was performed by homogenizing right hind paw tissues in separate parallel experiment. ${ }^{+} P<0.1$, significantly different from adjuvant-treated control $(n=3)$. 
were decreased in AW300 and AP5, though not with statistical significance.

The above findings clearly indicate that WOE possesses anti-arthritic activity in rats. In particular, the inhibition of IL-1 $\beta$ level by AW was notable. Thus, in order to evaluate the inhibitory mechanism of IL-1 $\beta$ production in the paw tissue, Western blot was performed using cellular lysate. Figure 2A shows that the levels of
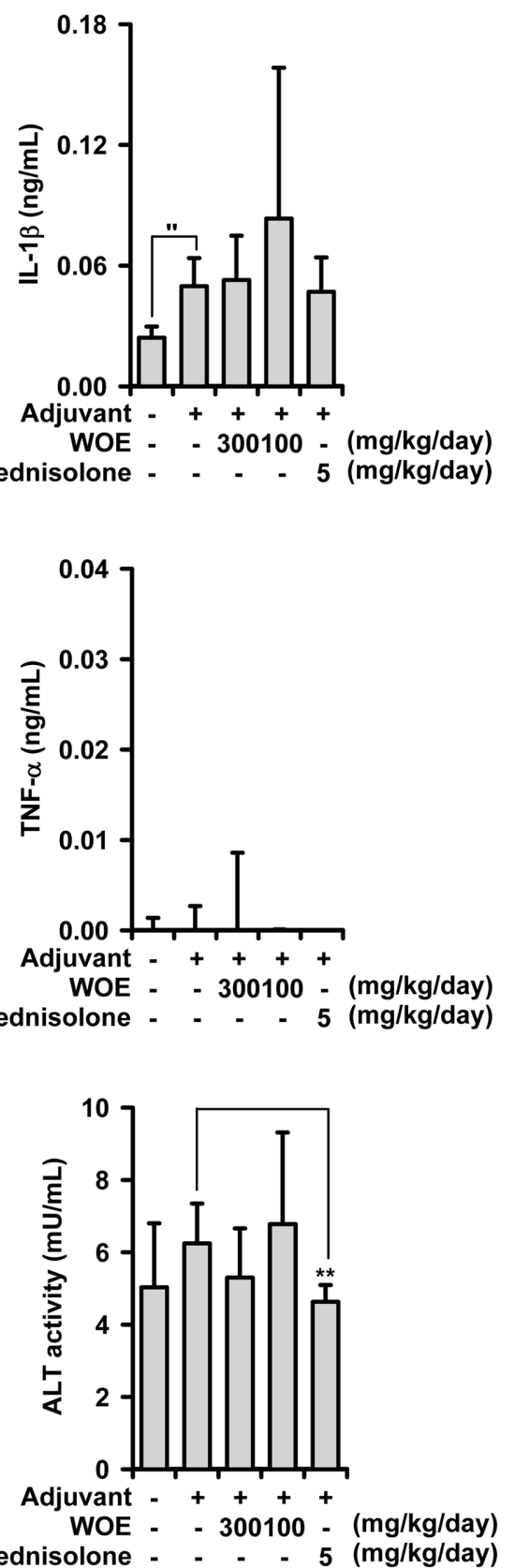

MAPKs expression were measured, and c-jun N-terminal kinase (JNK) in AC was found to be highly activated compared to the control group (C), while p38 MAPK and extracellular signal-regulated kinase (ERK) were rather weakly activated in AC. Under this condition, AW strongly reduced JNK activation, although the inhibition degree was not statistically significant. ERK activation was also inhibited, while p38 MAPK activation was not
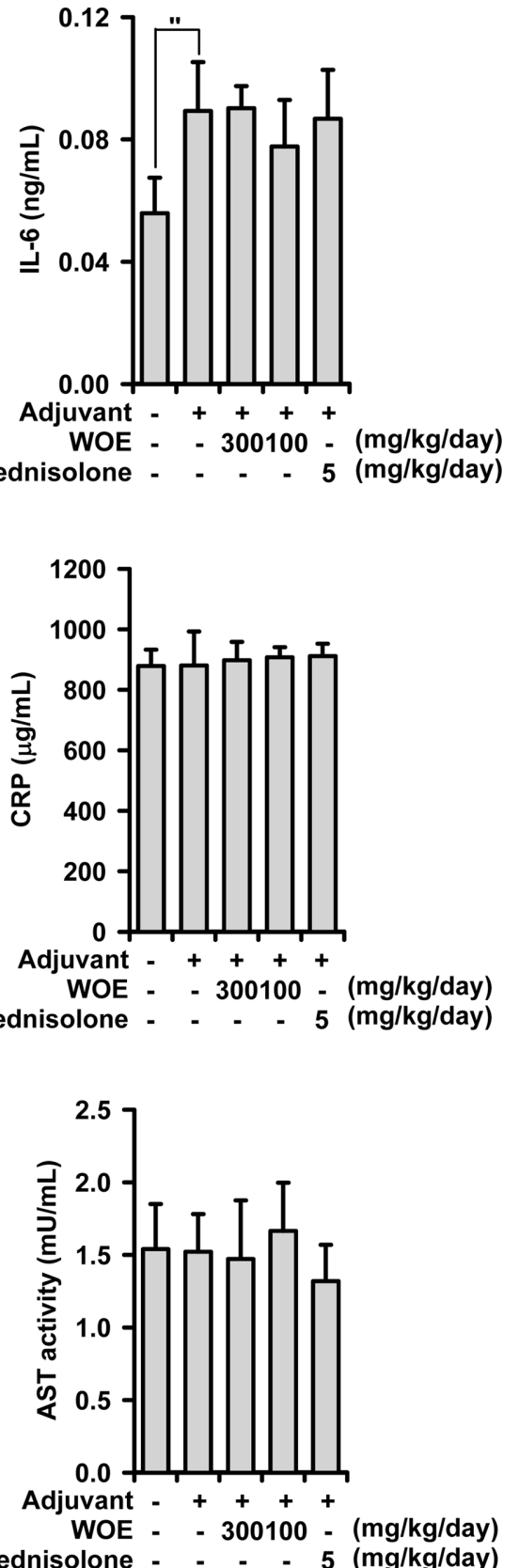

Fig. 3. Effects of WOE on pro-inflammatory molecules of the serum in adjuvant-induced arthritic rats. The amount of IL-1 $\beta$, IL-6, TNF$\alpha$, and C-reactive protein (CRP) and activity of ALT and AST in the serum of rats treated WOE (100 and $300 \mathrm{mg} / \mathrm{kg} / \mathrm{day})$ and prednisolone $(5 \mathrm{mg} / \mathrm{kg} /$ day $)$ were measured using ELISA kits. " $P<0.05$, significantly different from control; ${ }^{+} P<0.1,{ }^{*} P<0.05,{ }^{* *} P<$ 0.01 , significantly different from adjuvant-treated control. 
reduced by AW. The degrees of major nuclear transcription factors, NF- $\mathrm{kB}$ and activator protein-1 (AP-1), were also measured (Fig. 2B). NF- $\kappa \mathrm{B}$ was activated in $\mathrm{AC}$ compared to control group (C), while WOE treatment clearly inhibited NF-kB activation. On the other hand, the activation of c-Jun was not affected by adjuvant injection (AC). Thus it is suggested that IL- $1 \beta$ expression might be mediated, at least in part, by the inhibition of ERK and JNK/NF- $\mathrm{KB}$ activation.

To examine the effects of WOE on the concentrations of proinflammatory molecules in the serum of adjuvantinduced arthritis rats (systemic effect), the amount of proinflammatory cytokines, the concentration of Creactive protein (CRP), and ALT and AST activities in the isolated serum were measured using ELISA. It was found that adjuvant injection significantly increased IL-1 $\beta$ and IL-6 concentrations of the serum (AC), compared to the levels of the control group (C). However, TNF- $\alpha$, CRP, ALT, and AST levels were not affected (Fig. 3). Under this condition, WOE did not exert any significant inhibitory effect on the concentrations of all these molecules. Only slight reduction of ALT concentration was observed in AP5. All these results indicate that WOE did not affect the systemic inflammatory condition raised by adjuvant treatment. Thus it is suggested that WOE has no inhibitory effect on proinflammatory cytokines production, nor does it affect liver inflammatory response in the serum of AIA rats. WOE only affected the arthritic paw inflammatory parameters.

The present study has clearly shown that the water extract of $O$. elatus (WOE) possesses anti-arthritic activity in vivo. It is proved in an animal model of rheumatoid arthritis, AIA in rats. Part of the action mechanism was found to be the inhibition of MAPK/NF- $\mathrm{KB}$ signaling pathway. On the other hand, WOE did not affect systemic inflammation. WOE may be a new potential therapeutic against RA.

In the present investigation, anti-arthritic action was partly mediated by MAPK/NF- $\mathrm{kB}$ inhibitory mechanisms. In vitro anti-inflammatory action mechanism of WOE was identified in our unpublished experiment. Thus, the in vivo action mechanism is, at least in part, correlated with that of the in vitro effect.

$O$. elatus contains varieties of constituents that include essential oil, saponins, flavonoids, anthraquinones, polyacetylenes, and syringin. ${ }^{5}$ In particular, it was previously found that syringin orally-administered suppressed paw edema in rats and lung inflammation in mice..,11 Anthraquinones, including emodin and chrysophanol, have also been previously demonstrated to inhibit NO production in LPS-treated macrophages. ${ }^{12,13}$ In addition, oploxynes A and B, polyacetylenes, isolated from the stems of $O$. elatus inhibited LPS-induced NO production. ${ }^{14}$ Thus, all these compounds in WOE might contribute, at least in part, to anti-arthritic action in vivo.

Some plant extracts have been used to treat rheumatoid arthritis. For example, the anti-arthritic effects of Akebia quinata, Ephedra sinica, and Sophora subprostrata have been demonstrated. ${ }^{15}$ In addition, methanol extracts of cyathocline purpurea and costus speciosus showed antiarthritic effects in AIA rat models. ${ }^{16,17}$ Furthermore, studies on the anti-arthritic and anti-inflammatory effects of many plant extracts have been reported. Traditionally, $O$. elatus has long been used to treat a variety of health conditions, including arthritis, pain, and diabetes.

In conclusion, the water extract of the stems of $O$. elatus (WOE) is for the first time evaluated for its in vivo anti-arthritic effect. Against adjuvant-induced arthritis in rats, WOE showed significant inhibitory activity at $100-$ $300 \mathrm{mg} / \mathrm{kg} /$ day by oral administration. It showed antiarthritic inflammatory action and inhibited IL-1 $\beta$ production in the lesion, at least in part by interrupting MAPK/NF- $k B$ pathway. But it did not affect systemic inflammatory response. All these findings suggest that the water extract of $O$. elatus may be a potential therapeutic for treating the symptoms of rheumatoid arthritis, with less side-effect.

\section{Acknowledgements}

This study was financially supported of ' $R \& D$ Program for Forest Science Technology (Project No. 2017038A001919-BA01)' provided by Korea Forest Service (Korea Forestry Promotion Institute) and BK21-plus project from the Ministry of Education (Korea). Bioassay facility of New Drug Development Inst. (KNU) was used and greatly acknowledged.

\section{References}

(1) Crofford, L. J. Arthritis Res. Ther. 2013, 15, S2.

(2) Ong, C. K.; Lirk, P.; Tan, C. H.; Seymour, R. A. Clin. Med. Res. 2007, 5, 19-34.

(3) Kang, S.; Tanaka, T.; Kishimoto, T. Int. Immunol. 2015, 27, 21-29.

(4) Kumar, S.; Bhosle, D.; Janghel, A.; Deo, S.; Raut, P.; Verma, C.; Agrawal, M.; Amit, N.; Sharma, M.; Giri, T.; Tripathi, D. K.; Ajaz, A.; Alexander, A. Research J. Pharm. Tech. 2015, 8, 597-610.

(5) Shikov, A. N.; Pozharitskaya, O. N.; Makarov, V. G.; Yang, W. Z.; Guo, D. A. Chin. J. Nat. Med. 2014, 12, 721-729.

(6) Zhong Hua Ben Cao Commission. Chinese Materia Medica (Zhong Hua Ben Cao); Shanghai Science and Technology Press: China, 1999, pp 5026-5027.

(7) Zhang, S. C.; Wang, K. Z. Acta Pharm. Sin. 1980, 15, 81-85. 
(8) Qu, S. Y.; Jiang, X. L.; Wu, Y. J.; Wang, Y. H. Chin. Tradit. Herb. Drugs 1986, 17, 25-26.

(9) Kim, H. K.; Son, K. H.; Chang, H. W.; Kang, S. S.; Kim, H. P. Planta Med. 1999, 65, 465-467.

(10) Choi, J.; Shin, K. M.; Park, H. J.; Jung, H. J.; Kim, H. J.; Lee, Y. S.; Rew, J. H.; Lee, K. T. Planta Med. 2004, 70, 1027-1032.

(11) Zhang, A.; Liu, Z.; Sheng, L.; Wu, H. J. Surg. Res. 2017, 209, 252257.

(12) Kwon, K. S.; Lee, J. H.; So, K. S.; Park, B. K.; Lim, H.; Choi, J. S.; Kim, H. P. Phytother. Res. 2018, 32, 1537-1545.

(13) Zhu, T.; Zhang, W.; Feng, S. J.; Yu, H. P. Int. Immunopharmacol. 2016, 34, 16-24.

(14) Yang, M. C.; Kwon, H. C.; Kim, Y. J.; Lee, K. R.; Yang, H. O. J. Nat. Prod. 2010, 73, 801-805.
(15) Kim, S. Y.; Son, K. H.; Chang, H. W.; Kang, S. S.; Kim, H. P. Arch. Pharm. Res. 1997, 20, 313-317.

(16) Bihani, G. V.; Rojatkar, S. R.; Bodhankar, S. L. Biomed. Aging Pathol. 2014, 4, 197-206.

(17) Srivastava, S.; Singh, P.; Jha, K. K.; Mishra, G.; Srivastava, S.; Khosa, R. L. J. Ayurveda Integr. Med. 2012, 3, 204-208.

Received May 9, 2019 Revised September 10, 2019 Accepted September 10, 2019 